Pacific

Journal of

Mathematics

\title{
SMALL CONTRACTIONS OF SMOOTH VARIETIES
}

\author{
Gianluca Occhetta
}




\title{
SMALL CONTRACTIONS OF SMOOTH VARIETIES
}

\author{
Gianluca Occhetta
}

Let $\varphi: X \rightarrow W$ be a proper surjective map from a smooth complex projective variety $X$ to a normal variety $W$; if $\varphi$ has connected fibers and $-K_{X}$ is $\varphi$-ample, $\varphi$ is called a Fano-Mori contraction; if $\varphi$ is an isomorphism in codimension 2 , then $\varphi$ is called a small contraction.

In this paper we study Fano-Mori contractions with fibers covered by large families of rational curves. After some general results we specialize to the case of small contractions, giving a complete description of small contractions of fivefolds with smooth fibers and relatively spanned anticanonical bundle.

\section{Introduction.}

A contraction $\varphi: X \rightarrow W$ is a proper surjective map of normal varieties with connected fibers; if $X$ is a smooth complex projective $n$-dimensional variety, as we will assume throughout the paper, the contraction $\varphi$ is called Fano-Mori, or extremal if the anticanonical divisor $-K_{X}$ is $\varphi$-ample (for terminology and general properties of these maps see Section 2). Fano-Mori contractions of smooth varieties are defined by linear systems $\left|m\left(K_{X}+r L\right)\right|$, with $L$ a $\varphi$-ample line bundle, $m \gg 0$ and $r$ a positive integer; $K_{X}+r L$ is called a good supporting divisor of the contraction.

An important property of a Fano-Mori contraction is the existence of rational curves in its fibers, as proved by Mori [Mo1], [Mo2] for contractions of smooth varieties, and by Kawamata [Ka1], who extended the result to the log-terminal case. Studying families of rational curves with good properties (the so called unsplit families) on the fibers Ionescu and Wiśniewski proved the fiber locus inequality (Proposition 2.3.6), an inequality which involves the dimensions of the fibers, of the exceptional locus and of the ambient variety and the minimum anticanonical degree of the contracted curves. In particular, if $K_{X}+r L$ is a good supporting divisor of the contraction and $S^{\prime}$ is an irreducible component of a fiber of $\varphi$, we have

$$
\operatorname{dim} S^{\prime} \geq r+\operatorname{codim}(E(\varphi))-1 .
$$

The border case has been studied in $[\mathbf{A B W} 2,1.1]$, where the authors proved that the normalization of $S^{\prime}$ is a projective space; we will deal with the next 
case, giving a description of $S^{\prime}$ when $\operatorname{dim} S^{\prime}=r+\operatorname{codim}(E(\varphi))$; this is the content of Theorem 4.1.

This theorem is the starting point of the study of small contractions which we develop in the rest of the paper; our main result is Theorem 5.1, which gives a complete description of small contractions supported by $K_{X}+(n-4) L$ with smooth fibers and such that $-K_{X}$ is $\varphi$-spanned.

In particular we give a complete description of smooth fibers of small contractions of fivefolds with $-K_{X} \varphi$-spanned:

\begin{tabular}{|r|c|}
\hline$F$ & $N_{F / X}$ \\
\hline $\mathbb{P}^{3}$ & $\mathcal{O}_{\mathbb{P}^{3}}(-1) \oplus \mathcal{O}_{\mathbb{P}^{3}}(-1)$ \\
& $\mathcal{O}_{\mathbb{P}^{3}}(-2) \oplus \mathcal{O}_{\mathbb{P}^{3}}(-1)$ \\
$\mathbb{Q}^{3}$ & $\mathcal{O}_{\mathbb{Q}^{3}}(-1) \oplus \mathcal{O}_{\mathbb{Q}^{3}}(-1)$ \\
$\mathbb{P}^{2}$ & $\mathcal{O}_{\mathbb{P}^{2}} \oplus \mathcal{O}_{\mathbb{P}^{2}}(-1)^{\oplus 2}$ \\
\hline
\end{tabular}

Finally let us mention that, as a preparatory step for the Proof of Theorem 4.1, we prove that smooth fibers of any Fano-Mori contraction are rationally connected (see Corollary 3.6).

\section{Background material.}

2.1. Fano-Mori contractions. Let $X$ be a smooth complex projective variety; let $N_{1}(X)=\frac{\{1-\text { cycles }\}}{\equiv} \otimes \mathbb{R}, N^{1}(X)=\frac{\{\text { divisors }\}}{\equiv} \otimes \mathbb{R}$, and $\overline{N E(X)}=$ $\overline{\text { \{effective 1-cycles }\}}$; the last is a closed cone in $N_{1}(X)$; we also set $\rho(X)=$ $\operatorname{dim}_{\mathbb{R}} N^{1}(X)$.

Suppose that $K_{X}$ is not nef, that is there exists an effective curve $C$ such that $K_{X} . C<0$; by the Kawamata-Shokurov Contraction Theorem there exist a normal projective variety $Y$ and a surjective morphism $\varphi: X \rightarrow Y$ with connected fibers which contracts curves whose numerical class lies in a face in the negative part of the cone $\overline{N E(X)}$ and such that $-K_{X}$ is $\varphi$-ample.

Definition 2.1.1 ([AW2, (1.0)]). The map $\varphi$ is called a Fano-Mori contraction (or an extremal contraction); the set $F$ is an extremal face, while a nef Cartier divisor $H$ s.t. $F=H^{\perp} \cap \overline{N E(X)}$ is a good supporting divisor for the $\operatorname{map} \varphi$ (or the face $F$ ). The contraction is of fiber type if $\operatorname{dim} Y<\operatorname{dim} X$, otherwise it is birational; a birational contraction which is an isomorphism in codimension 2 is called a small contraction. If $\operatorname{dim}_{\mathbb{R}} F=1$ the face $F$ is called an extremal ray, while $\varphi$ is called an elementary contraction.

Definition 2.1.2. Let $\varphi: X \rightarrow W$ be a small elementary contraction; the flip of $\varphi$ is a commutative diagram 


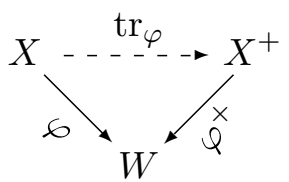

where $\varphi^{+}: X^{+} \rightarrow W$ is a birational morphism from a normal projective variety with only terminal singularities such that the canonical divisor is $\varphi^{+}$-ample and $X^{+}$is isomorphic to $X$ in codimension 1 via $\operatorname{tr}_{\varphi}$.

Remark 2.1.3. We have also (see [Mo2]) that if $X$ has an extremal ray $R$ then there exists a rational curve $C$ on $X$ such that $0<-K_{X} \cdot C \leq n+1$ and $R=\mathbb{R}_{+}[C]:=\left\{D \in N E(X): D \equiv \lambda C, \lambda \in \mathbb{R}^{+}\right\}$. A rational curve in $R$ whose intersection number with the anticanonical bundle is minimal is called an extremal curve.

2.2. Apollonius method. In the study of Fano-Mori contractions it is often very useful to apply a kind of inductive method, called Apollonius method, slicing the fibers.

Local setup. We assume that $\varphi: Y \rightarrow W$ is a Fano-Mori contraction of a smooth variety $Y$ onto a normal variety $W$; we choose a fiber $F$ and an open affine subset $Z \subset W$ such that $\varphi(F) \in Z$. Let $X=\varphi^{-1}(Z)$; we will call $\varphi: X \rightarrow Z$ a local contraction around $F$.

If $L$ is a $\varphi$-ample line bundle and $r$ a rational number such that $K_{X}+r L$ is trivial on the fibers of $\varphi$, then $K_{X}+r L$ is a good supporting divisor for the local contraction $\varphi$.

The idea of the Apollonius method is to slice $X$ in order to obtain a new variety $X^{\prime}$ with a contraction $\varphi: X^{\prime} \rightarrow Z^{\prime}$ with smaller fibers, which is easier to study, and then "ascend" the properties of $X^{\prime}$ to $X$. The first step is given by the following two lemmata:

Lemma 2.2.1 ([AW1, Lemma 2.6]; (horizontal slicing)). Suppose that $\varphi$ : $X \rightarrow W$ is a local contraction supported by $K_{X}+r L$, and let $X^{\prime}$ be a general divisor in the linear system $|L|$. Then, outside of the base locus of $|L|$, the singularities of $X^{\prime}$ are not worse than those of $X$ and any section of $L$ on $X^{\prime}$ extends to $X$.

Moreover, if we set $\varphi^{\prime}:=\varphi_{\mid X^{\prime}}$ and $L^{\prime}=L_{X^{\prime}}$, then $K_{X^{\prime}}+(r-1) L^{\prime}$ is $\varphi^{\prime}$-trivial. If $r \geq 1+\epsilon(\operatorname{dim} X-\operatorname{dim} Z)$ then $\varphi^{\prime}$ is a contraction, i.e., has connected fibers.

Lemma 2.2.2 ([AW1, Lemma 2.5]; (vertical slicing)). Assume that $\varphi: X$ $\rightarrow W$ is a local contraction supported by $K_{X}+r L$ and let $X^{\prime \prime} \subset X$ be a nontrivial divisor defined by a global function $h \in H^{0}\left(X, K_{X}+r L\right)=$ $H^{0}\left(X, \mathcal{O}_{X}\right)$; then, for a general choice of $h, X^{\prime \prime}$ has singularities not worse than those of $X$ and any section of $L$ on $X^{\prime}$ extends to $X$. 
We will be interested in the study of normal bundles, so it is very important to know how to ascend their properties. The following construction was developed in [AW2]:

Let $\varphi: X \rightarrow Z$ be a Fano-Mori or crepant contraction of a smooth variety, $L$ an ample line bundle on $X, F=\varphi^{-1}(z)$ a fiber of $\varphi$ which is locally complete intersection and $X^{\prime} \in|L|$ a normal divisor which does not contain any component of $F$. Considering the embeddings $F^{\prime}=F \cap X^{\prime} \subset F \subset X$ and $F^{\prime} \subset X^{\prime} \subset X$ we obtain

$$
N_{F^{\prime} / X}=N_{F^{\prime} / X^{\prime}} \oplus L_{F^{\prime}}=\left(N_{F / X}\right)_{\mid F^{\prime}} \oplus L_{F}
$$

and therefore $N_{F^{\prime} / X^{\prime}}=\left(N_{F / X}\right)_{\mid F^{\prime}}$; this fact will be used together with the following:

Lemma 2.2.4 ([AW2, 5.7.2]). If the bundle $N_{F^{\prime} / X^{\prime}}^{*}$ is spanned by functions of $\Gamma\left(X^{\prime}, \mathcal{O}_{X^{\prime}}\right)$ at a point $x \in F^{\prime}$, then the bundle $N_{F / X}^{*}$ is spanned at $x$ by functions of $\Gamma\left(X, \mathcal{O}_{X}\right)$. If $N_{F^{\prime} / X^{\prime}}^{*}$ is spanned by functions from $\Gamma\left(X^{\prime}, \mathcal{O}_{X^{\prime}}\right)$ everywhere on $F^{\prime}$, then $N_{F / X}^{*}$ is nef.

2.3. Unsplit families of rational curves. Throughout this section our main reference is $[\mathbf{K o}]$, with which our notation is coherent.

Let $X$ be a smooth variety, and $x \in X$ a point; consider the schemes $\operatorname{Hom}\left(\mathbb{P}^{1}, \mathrm{X}\right)$, parametrizing morphisms from $\mathbb{P}^{1}$ to $X$, and $\operatorname{Hom}\left(\mathbb{P}^{1}, \mathrm{X} ; 0 \rightarrow\right.$ $\mathrm{x}$ ), parametrizing morphisms sending $0 \in \mathbb{P}^{1}$ to $x \in X$.

Let $V \subset \operatorname{Hom}\left(\mathbb{P}^{1}, \mathrm{X}\right)$ be a closed irreducible subvariety; we will call $V$ a family of morphisms or, by abuse, a family of rational curves on $X$.

The image of $V$ via the restriction of the evaluation morphism $F: \mathbb{P}^{1} \times$ $\operatorname{Hom}\left(\mathbb{P}^{1}, \mathrm{X}\right) \rightarrow \mathrm{X}$ will be denoted by $\operatorname{Locus}(\mathrm{V})$ and called locus of the family; finally we will denote by $\operatorname{Locus}(\mathrm{V}, 0 \rightarrow \mathrm{x})$ the locus of $V \cap \operatorname{Hom}\left(\mathbb{P}^{1}, \mathrm{X} ; 0 \rightarrow \mathrm{x}\right)$, i.e., the locus of the curves in the family which pass through $x$, and by $\operatorname{deg}_{-\mathrm{K}}(\mathrm{V})$ the anticanonical degree of a general curve in the family.

Definition 2.3.1. A family of rational curves is called unsplit if the image of $V$ in $\operatorname{Chow}(X)$ is closed.

Remark 2.3.2 ([Ko, IV.2.2]). Let $W \subset \operatorname{Chow}(X)$ be the image of $V$ and $\left[C_{g}\right]$ be the cycle corresponding to the generic point of $W$; the points in $\bar{W} \backslash W$ correspond to degenerations of $\left[C_{g}\right]$ into reducible cycles.

Example 2.3.3. The family of deformations of an extremal rational curve $C$ is an unsplit family: If $C$ degenerates into a reducible cycle, the components must belong to the ray $R$ generated by $C$, since $R$ is extremal; but in $R$ the curve $C$ has the minimal intersection with the anticanonical bundle, hence this is impossible. 
Now we recall some results which give useful properties of unsplit families that we will use in the rest of the paper.

Lemma 2.3.4 (see [Wi1, Appendix]). Let $X$ be a smooth variety, $V$ an unsplit family of rational curves on $X$ and fix $x \in \operatorname{Locus}(\mathrm{V})$; every morphism $h: \operatorname{Locus}(\mathrm{V}, 0 \rightarrow \mathrm{x}) \rightarrow \mathrm{Z}$ is either finite-to-one or takes $\operatorname{Locus}(\mathrm{V}, 0 \rightarrow \mathrm{x})$ to a point.

Proposition 2.3.5 ([Ko, IV.2.6]). Let $X$ be a smooth and proper variety and $V$ an unsplit family of rational curves. Then, for $x \in \operatorname{Locus}(\mathrm{V})$ :

1) $\operatorname{dim} X+\operatorname{deg}_{-\mathrm{K}}(\mathrm{V}) \leq \operatorname{dim} \operatorname{Locus}(\mathrm{V})+\operatorname{dim} \operatorname{Locus}(\mathrm{V}, 0 \rightarrow \mathrm{x})+1=\operatorname{dim} \mathrm{V}$

2) $\operatorname{dim} X+\operatorname{deg}_{-\mathrm{K}}(\mathrm{V}) \leq 2 \operatorname{dim} \operatorname{Locus}(\mathrm{V})+1$

3) $\operatorname{deg}_{-\mathrm{K}}(\mathrm{V}) \leq \operatorname{dim} \operatorname{Locus}(\mathrm{V}, 0 \rightarrow \mathrm{x})+1$.

This last proposition, in case of the unsplit family of deformations of an extremal rational curve, gives the fiber locus inequality:

Proposition 2.3.6 ([Io], [Wi2]). Let $\varphi$ be a Fano-Mori contraction of $X$ and let $E=E(\varphi)$ be the exceptional locus of $\varphi$ (if $\varphi$ is of fiber type then $E:=X)$; let $S$ be an irreducible component of a (nontrivial) fiber $F$. Then

$$
\operatorname{dim} S+\operatorname{dim} E \geq \operatorname{dim} X+l-1
$$

where

$$
l=\min \left\{-K_{X} \cdot C: C \text { is a rational curve in } S\right\} .
$$

If $\varphi$ is the contraction of a ray $R$, then $l$ is called the length of the ray.

The projective space can be characterized as a variety covered by a large unsplit family of low degree rational curves:

Theorem 2.3.7 ([ABW2, 1.1]). Let $X$ be a normal projective variety, $L \in$ $\operatorname{Pic}(X)$ and $V$ an unsplit family of rational curves on $X$ such that, for a general $x \in X$, we have $\overline{\operatorname{Locus}(\mathrm{V}, 0 \rightarrow \mathrm{x})}=X$ and $L . C=1$ for a curve $C$ of $V$; then $(X, L) \simeq\left(\mathbb{P}^{n}, \mathcal{O}_{\mathbb{P}}(1)\right)$.

Theorem 2.3.8 ([Ke, Theorem 3.6]). Let $X$ be a normal projective variety, $L \in P i c(X)$ and $V$ an unsplit family of rational curves on $X$ such that, for a general $x \in X$, we have $\overline{\operatorname{Locus}(\mathrm{V}, 0 \rightarrow \mathrm{x})}=X$ and L.C $=2$ for a curve $C$ of $V$; then $(X, L) \simeq\left(\mathbb{P}^{n}, \mathcal{O}_{\mathbb{P}}(2)\right)$.

\section{Finding transverse rational curves.}

In this section we will prove the following:

Proposition 3.1. Let $\varphi: X \rightarrow W$ be a Fano-Mori contraction of a smooth variety, and let $F$ be a fiber of $\varphi$. Let $\pi: U \rightarrow Z$ be a surjective proper morphism from an open dense subset of $F$ onto a quasi projective variety $Z$ of positive dimension. Then for any point $z \in Z$ there exists a rational curve on $F$ which meets $\pi^{-1}(z)$ but is not contracted by $\pi$. 
This result grew out of reading [KoMiMo] via the following:

Remark 3.2. Let $\varphi: X \rightarrow W$ be a Fano-Mori contraction of a smooth variety, and let $F$ be a fiber of $\varphi$. The numerical class of any curve in $F$ is contained in the extremal face contracted by $\varphi$, and the same happens for the deformations of such curves. In particular, the deformations of a curve in the fiber are contained in the exceptional locus, and the pointed deformations of a curve in the fiber are contained in the fiber itself.

For the convenience of the reader, we recall some definitions and results from [KoMiMo]:

Definition 3.3. Let $X, Y$ and $Z$ be irreducible schemes. Let $U \subset X$ be an open dense subset, $\pi: U \rightarrow Z$ a morphism, $f: Y \rightarrow X$ with $f(Y)$ meeting $U$. By a relative deformation of $f$ over $Z$, parametrized by a connected punctured scheme $(S, 0)$ with a base subscheme $B \subset Y$, we mean a morphism

$$
F=\left\{f_{s}\right\}: Y \times S \rightarrow X
$$

which satisfies the following conditions:

1) $f_{0}=f$

2) $F_{\mid B \times S}=\left(f \circ p r_{Y}\right)_{\mid B \times S}$

3) $\pi f_{s}=\pi f$ for every $s \in S$.

If we are considering $Y$ to be a curve of positive genus, we can replace our original morphism with a new one which has no relative deformations over $Z$.

Proposition 3.4 ([KoMiMo, 2.4]). Assume that there is an open subset $U \subset X$ such that $\pi_{\mid U}$ is a proper morphism over an open subset of $Z$. Let $f: Y \rightarrow X$ be a morphism of a curve of positive genus. If $Z$ is not a single point, then there exists a morphism $f^{\prime}: Y \rightarrow X$ such that the following hold:

1) $\pi f^{\prime}=\pi f$

2) $\operatorname{Hom}_{\mathrm{Z}}\left(\mathrm{Y}, \mathrm{Z} ; \mathrm{f}^{\prime}, \mathrm{B}\right)$ is a zero-dimensional scheme, when $B$ is nonempty.

We are going to apply this result to fibers of Fano-Mori contractions using the following:

Lemma 3.5 ([KoMiMo, Lemma 2.5]). Let $\pi: X \rightarrow Z$ be a dominant rational map between projective varieties. Let $H$ and $D$ be ample divisors on $X$ and $Z$, respectively. Then there exists a constant $\alpha$ which depends only on $\pi: X \rightarrow Z, H$ and $D$ such that

$$
\operatorname{deg} f^{*} H \geq \alpha \operatorname{deg}(\pi f)^{*} D
$$

for any smooth projective curve $Y$ and any morphism $f: Y \rightarrow X$ whose image meets the domain $U$ on which $\pi$ is defined.

Proof of Proposition 3.1. The proof follows the line of proof of [KoMiMo, Theorem 2.1], using the fact that, in view of Remark 3.2: 
- We can bound the dimension of the deformation space of a curve $C$ in $F$ using $-K_{X} . C$ instead of $-K_{F} \cdot C$.

- $-K_{X \mid F}$ is ample.

Step I. Char $\mathbb{K}>0$.

The divisor $-K_{X \mid F}$ is ample on $F$; fix an ample divisor $D$ on $Z$ and let $\alpha$ be the constant such that

$$
\operatorname{deg} f^{*}\left(-K_{X \mid F}\right) \geq \alpha \operatorname{deg}(\pi f)^{*} D
$$

whose existence is assured by Lemma 3.5. Choose a point $z \in Z$ and a smooth curve $Y \subset F$ which intersects $\pi^{-1}(z)$; fix a point $P_{0}$ in $Y \cap \pi^{-1}(z)$ and choose a Frobenius morphism $f: Y \rightarrow Y \subset F$ such that $\operatorname{deg} \pi f>\frac{n}{\alpha} g(Y)$.

By Proposition 3.4 we can replace $f$ by $f^{\prime}: Y \rightarrow X$ such that

$$
\operatorname{deg} \pi f^{\prime}=\operatorname{deg} \pi f>\frac{n}{\alpha} g(Y)
$$

and $f^{\prime}$ has no relative deformations over $Z$ with base point $P=f^{-1}\left(P_{0}\right)$. By the first condition we have

$$
\chi\left(Y, \mathcal{I}_{P_{0}} f^{\prime *} T_{X}\right)=\operatorname{deg} f^{\prime *}\left(-K_{X}\right)-g(Y) \operatorname{dim} X>0
$$

so there exist absolute deformations of $Y_{0}$ and hence a rational curve through $P_{0}$; since $f^{\prime}$ has no relative deformations over $Z$, its deformations induce nontrivial deformations of $\pi f^{\prime}$ on $Z$, so there exists a rational curve on $F$ through $P_{0}$ which is mapped to a rational curve on $Z$; then, deforming this curve, we can split it into a union of rational curves of degree $\leq \operatorname{dim} X+1$, one of which meets $\pi^{-1}(z)$.

Step II. Lifting to characteristic zero.

This is a standard construction, since the rational curve we found has bounded degree with respect to the ample divisor $-K_{X \mid F}$.

The existence of transverse rational curves was proved in [KoMiMo] as a step in the proof of rational connectedness of smooth Fano varieties; an analogous result holds for smooth fibers of Fano-Mori contractions, while it fails if we consider singular fibers.

Corollary 3.6. Let $\varphi: X \rightarrow W$ be a Fano-Mori contraction of a smooth variety and $F$ a smooth fiber of $\varphi$. Then $F$ is rationally connected.

Proof. Consider the maximal rationally chain connected fibration $F-Z$ (see [Ko, Theorem 5.2]); if $Z$ is not a point there exists a rational curve passing through a point of a very general fiber of $\rho$ which is not contracted by $\rho$ and this is a contradiction, by the maximality of $\rho$ (see $[\mathbf{K o}, 5.2 .1]$ ). Thus $Z$ is a point and $F$ is rationally chain connected, but, in characteristic zero, rational chain connectedness is equivalent to rational connectedness. 
Corollary 3.7. Let $\varphi: X \rightarrow W$ be a Fano-Mori contraction of a smooth variety and $F$ a smooth fiber of $\varphi$. Then

$$
H^{i}\left(F, \mathcal{O}_{F}\right)=0 \quad \text { for } i \geq 1 .
$$

Example 3.8. A singular fiber of a Fano-Mori contraction which is not rationally connected (suggested by Jaroslaw Wiśniewski).

Let $W$ be the projectivization of the linear system $\left|\mathcal{O}_{\mathbb{P}^{3}}(3)\right|, X \subset \mathbb{P}^{3} \times W$ the incidence variety and $p$ the projection onto $\mathrm{W}$; it is easy to check that $-K_{X}$ is $p$-ample (it restricts to $\mathcal{O}(1)$ on the fibers of $p$ ), so that $p$ is a FanoMori contraction; among its fibers there is the cubic singular surface given by the cone over an elliptic curve, which is not rationally connected.

\section{Deformations of curves and fibers of Fano-Mori contractions.}

In this section we will prove the following:

Theorem 4.1. Let $\varphi: X \rightarrow W$ be a Fano-Mori contraction of a smooth variety, supported by $K_{X}+r L$, Let $S^{\prime}$ be any component of a nontrivial fiber $F=\varphi^{-1}(w)$, let $S$ be its normalization and denote again by $L$ the pullback of $L$ to $S$. If

$$
\operatorname{dim} S=r+\operatorname{codim}(E(\varphi))
$$

then either the Fujita $\Delta$-genus of $(S, L)$ is zero or $S$ is singular and has a desingularization which is a $\mathbb{P}^{s-1}$-bundle over a smooth curve.

Moreover, if $S^{\prime}$ has rational singularities then only the first possibility can occur.

Proof. Let $C \subset S$ be an extremal rational curve, $V$ the unsplit family of deformations of $C$ and $x$ a general point in Locus $(\mathrm{V})$; we have

$$
\begin{aligned}
& n+r=\operatorname{dim} E(\varphi)+\operatorname{dim} S \\
& \geq \operatorname{dim} \operatorname{Locus}(\mathrm{V})+\operatorname{dim} \operatorname{Locus}(\mathrm{V}, 0 \rightarrow \mathrm{x}) \geq \mathrm{n}+\mathrm{r}-1,
\end{aligned}
$$

whence $\operatorname{Locus}(\mathrm{V})=\mathrm{E}(\varphi)$ and $\operatorname{dim} \operatorname{Locus}(\mathrm{V}, 0 \rightarrow \mathrm{x})=\mathrm{s}, \mathrm{s}-1$.

Let $S$ be the normalization of $S^{\prime}$; normalizing also the family and its graph we obtain a new unsplit family $\mathcal{V}$ on $S$.

If $\operatorname{deg}_{-\mathrm{K}}(\mathcal{V})=\mathrm{r}$ then the curves in this family are lines with respect to $L$, otherwise we have $r=1$ and $\operatorname{deg}_{-\mathrm{K}}(\mathcal{V})=2$; in this last case, applying Theorem 2.3.8 we get $(S, L) \simeq\left(\mathbb{P}^{s}, \mathcal{O}_{\mathbb{P}}(2)\right)$.

Let $\theta: M \rightarrow S$ be a desingularization of $S, L_{M}=\theta^{*} L$, and $m$ the inverse image of a smooth point $e$ of $S$. The curves of $\mathcal{V}$ passing through $e$, since they are not entirely contained in the singular locus of $S$, can be lifted to curves on $M$ passing through the point $m$.

We claim that there is a family $\mathcal{V}_{M}$ of these lifted up curves which has degree one with respect to $L_{M}$, and covers a dense subset of $M$. 
Indeed we know that a neighbourhood of $m$ is covered by these curves, so, if such a family did not exist, the neighbourhood would be contained in a countable sum of nowhere dense subsets of $M$, obtained by deforming each of the lifted up curves, a contradiction.

By construction, for the family $\mathcal{V}_{M}$ we have

$$
\begin{aligned}
& \operatorname{dim} \operatorname{Locus}\left(\mathcal{V}_{\mathrm{M}}\right)=\mathrm{s} \\
& \operatorname{dim} \operatorname{Locus}\left(\mathcal{V}_{\mathrm{M}}, 0 \rightarrow \mathrm{m}\right) \geq \mathrm{s}-1 .
\end{aligned}
$$

Let ev $: \mathcal{V}_{M} \times \mathbb{P}^{1} \rightarrow M$ be the evaluation map and let $f: \mathbb{P}^{1} \rightarrow M$ be a general member of the family; we have (cf. [Ko, II.3])

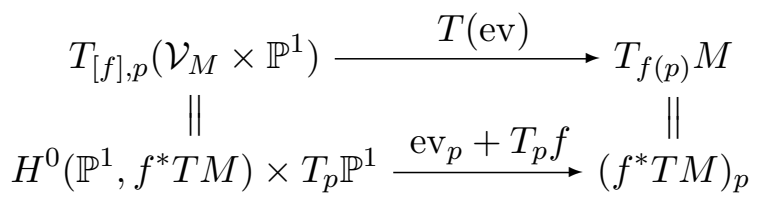

and

$$
\begin{gathered}
T_{[f], p}\left(\left(\mathcal{V}_{M}, 0 \rightarrow m\right) \times \mathbb{P}^{1}\right) \stackrel{T(\mathrm{ev})}{\longrightarrow} T_{f(p)} M \\
H^{0}\left(\mathbb{P}^{1}, f^{*} T M \otimes I_{m}\right) \times T_{p} \mathbb{P}^{1} \stackrel{\mathrm{ev}_{p}+T_{p} f}{\longrightarrow}\left(f^{*} T M\right)_{p} .
\end{gathered}
$$

In both cases we have $\operatorname{rk}(T(\mathrm{ev}))=\operatorname{rk}\left(\mathrm{ev}_{p}\right)$ so, by the information on the dimensions of the loci, we deduce

$$
f^{*}(T M) \simeq \oplus \mathcal{O}_{\mathbb{P}^{1}}\left(a_{i}\right)
$$

with $a_{1} \geq 2, \quad a_{i} \geq 1 \quad$ for $\quad i=2, \ldots s-1, \quad a_{s} \geq 0$, implying

$$
\left(K_{M}+t L_{M}\right) . C<0 \quad \text { if } t<s .
$$

So, since the family covers a dense subset of $M$, we have the following vanishing results:

$$
\begin{aligned}
h^{s}\left(M,-t L_{M}\right)=h^{0}\left(M, K_{M}+t L_{M}\right)=0 & \text { for } t \leq s-1 \\
h^{i}\left(M, \mathcal{O}_{M}\right)=h^{0}\left(M, \wedge^{i} T M^{*}\right)=0 & \text { for } i \geq 2 .
\end{aligned}
$$

Case $1 . \quad h^{1}\left(M, \mathcal{O}_{M}\right)=0$.

By the Kawamata-Viehweg vanishing theorem we have

$$
h^{i}\left(M,-t L_{M}\right)=0 \text { for } i<s, t>0 .
$$

Combining (4.2), (4.3) and (4.4) we see that the Hilbert polynomial of $L_{M}$, $\chi(t)=\chi\left(M, t L_{M}\right)$, vanishes for $t=-1,-2, \ldots,-s+1$, and $\chi\left(M, \mathcal{O}_{M}\right)=1$, 
so we can write the polynomial in the form

$$
\chi\left(M, t L_{M}\right)=\frac{d}{s !}\left(\prod_{k=1}^{s-1}(t+k)\right)\left(t+\frac{s}{d}\right) .
$$

This implies that $h^{0}\left(M, L_{M}\right)=\chi(1)=s+d$ and then, computing the $\Delta$-genus of the quasi-polarized variety $\left(M, L_{M}\right)$,

$$
\Delta\left(M, L_{M}\right)=\operatorname{dim} M+\operatorname{deg} L_{M}-h^{0}\left(M, L_{M}\right)=s+d-(s+d)=0 .
$$

Using [Fu, Theorem 1.1] we get a commutative diagram

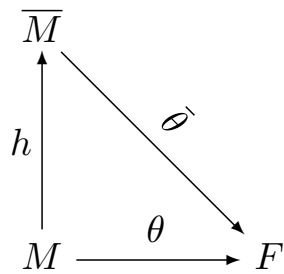

where $\bar{M}$ is a smooth variety and $L_{\bar{M}}$ is a very ample line bundle on $\bar{M}$ such that $\left(\bar{M}, L_{\bar{M}}\right)$ has $\Delta$-genus zero, so this is the case also for $\left(S, L_{S}\right)$.

Case $2 . \quad h^{1}\left(M, \mathcal{O}_{M}\right) \neq 0$.

Suppose that $H^{1}\left(M, \mathcal{O}_{M}\right)$ does not vanish and consider the Albanese map of $M, \alpha: M \rightarrow \operatorname{Alb}(M)$; since we know that $\overline{\operatorname{Locus}\left(\mathcal{V}_{\mathrm{M}}\right)}=M$ and that $\operatorname{dim} \operatorname{Locus}\left(\mathcal{V}_{\mathrm{M}}, 0 \rightarrow \mathrm{x}\right) \geq \mathrm{s}-1$, the image of the Albanese map of $M$, $\alpha(M) \subset \operatorname{Alb}(M)$ must be a nonsingular curve $B$.

Let $G$ be the generic fiber of $\alpha ; G$ is a quasi polarized variety of dimension $s-1$ covered by a family of rational curves whose dimension at the generic point is $s-1$. We have

$$
h^{s-1}\left(G,-t L_{G}\right)=h^{0}\left(f, K_{G}+t L_{G}\right)=0 \quad \text { for } t \leq s-1 .
$$

So, since $G$ is a desingularization of $\widetilde{G}$, the normalization of $\theta(G)$, by $[\mathbf{F u}$, Theorem 2.2] we have that $\widetilde{G}$ is a projective space $\mathbb{P}^{s-1}$.

Consider the map $\Theta: M \rightarrow S \times B$ induced by $\theta$, and the image $\Theta(M)$ of $M$, with the maps $p_{1}: \Theta(M) \rightarrow S$ and $p_{2}: \Theta(M) \rightarrow B$ induced by the projections of $S \times B$ on the factors:

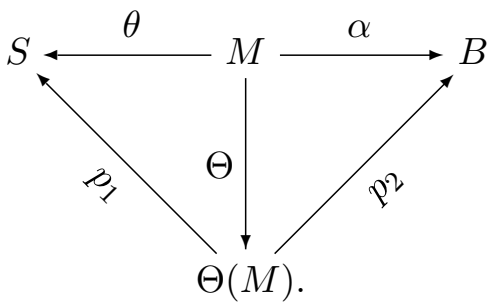


Let $\widetilde{M}$ be the normalization of $\Theta(M)$; we have the following commutative diagram:

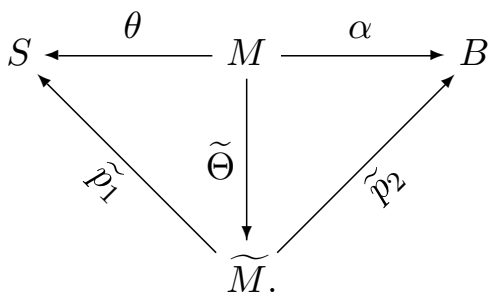

The generic fiber of $\widetilde{p_{2}}$, which is a normal variety, is $\mathbb{P}^{s-1}$ since $\widetilde{G}$ is so: The line bundle $L_{\widetilde{M}}=\widetilde{p}_{1}^{*} L$ is ample on the fibers of $\widetilde{p_{2}}$, so, up to replace it with $L_{\widetilde{M}}^{\prime}=L_{\widetilde{M}}+{\widetilde{p_{2}}}^{*} H$ where $H$ is ample on $B$, we can assume it is ample; then we argue as in [BS, Proposition 3.2.1] to obtain that $\left(\widetilde{M}, L_{\widetilde{M}}\right)$ is a $\mathbb{P}^{s-1}$-bundle over $B$; note that we can disregard the assumptions on the singularities of $\widetilde{M}$ since $\widetilde{p}_{2}$ is flat.

The last assertion follows from the fact that, in case of rational singularities, we can consider the Albanese map of $S^{\prime}$ itself and obtain that, in Case 2 of the Proof, $S^{\prime}$ is a $\mathbb{P}^{s-1}$-bundle over asmooth curve $B$, which has to be rational by Corollary 3.7 .

Remark 4.5. In the case of elementary divisorial contractions it is possible to replace the assumption $\operatorname{dim} S=r+\operatorname{codim}(E(\varphi))$ with $\operatorname{dim} S=l(\varphi)+$ codim $(E(\varphi))$, avoiding any assumption on $L$; in fact one can prove that $K_{X}-r E(\varphi)$ is a good supporting divisor for $\varphi$ (see [AO1]).

\section{Small contractions.}

In the second part of the paper, keeping Theorem 4.1 as a starting point, we will deal with small contractions. It is well-known that (locally) the only small contraction of smooth varieties of dimension four is the Kawamata small contraction $[\mathbf{K a 2}]$, i.e., the contraction of a projective plane with normal bundle $\mathcal{O}_{\mathbb{P}^{2}}(-1)^{\oplus 2}$; this contraction is supported by $K_{X}+L$, and one natural generalization of this situation is the study of contractions supported by $K_{X}+(n-3) L$ on a smooth $n$-fold; this was done in [ABW2].

The next step is the study of small contractions of smooth varieties of dimension five; this contractions can be supported either by $K_{X}+2 L$, and this situation falls in the case treated in [ABW2], or by $K_{X}+L$; this (or more generally the case of small contractions of $n$-folds supported by $\left.K_{X}+(n-4) L\right)$ will be the object of our study. Our results are summarized in the following:

Theorem 5.1. Let $K_{X}+(n-4) L$ be a good supporting divisor of an elementary small contraction $\varphi: X \rightarrow Z$, let $F$ be a smooth fiber of $\varphi$ and 
$N=N_{F / X}$ its normal bundle. If $L$ is $\varphi$-spanned then one of the following possibilities occurs:

\begin{tabular}{|r|l|l|}
\hline$n$ & \multicolumn{1}{|c|}{$F$} & \multicolumn{1}{|c|}{$N_{F / X}$} \\
\hline 5 & $\mathbb{P}^{3}$ & $\mathcal{O}_{\mathbb{P}}(-1) \oplus \mathcal{O}_{\mathbb{P}^{3}}(-1)$ \\
$\geq 5$ & $\mathbb{P}^{n-2}$ & $\mathcal{O}_{\mathbb{P}}(-2) \oplus \mathcal{O}_{\mathbb{P}}(-1)$ \\
$\geq 5$ & $\mathbb{Q}^{n-2}$ & $\mathcal{O}_{\mathbb{Q}}(-1) \oplus \mathcal{O}_{\mathbb{Q}}(-1)$ \\
$\geq 5$ & $\mathbb{P}^{n-3}$ & $\mathcal{O}_{\mathbb{P}} \oplus \mathcal{O}_{\mathbb{P}}(-1)^{\oplus 2}$ \\
\hline
\end{tabular}

Moreover, the formal neighborhood of $F$ in $X$ is determined uniquely and it is the same as the formal neighborhood of the zero section of the total space of the bundle $N_{F / X}^{*}$, and the flip of $\varphi$ exists.

The rest of the section is devoted to the proof of this theorem. We will proceed in several steps.

5.1. Geometric fiber. First of all we present another deformation argument which will help us to refine the description of the fibers given in Theorem 4.1 in the case of small contractions:

Proposition 5.1.1. Let $\varphi: X \rightarrow Z$ be a Fano-Mori elementary small contraction of a smooth variety supported by $K_{X}+(n-4) L$, and let $F$ be a fiber of $\varphi$. Then $F$ cannot be a $\mathbb{P}$-bundle over a rational curve.

Proof. By Proposition 2.3.6 the dimension of $F$ is $n-2$ or $n-3$; in the latter case the normalization of $F$ is a projective space by $[\mathbf{A B W} 2,1.1]$, so we have to deal only with the case $\operatorname{dim} F=n-2$.

Suppose that $F$ is a $\mathbb{P}^{n-3}$-bundle over a rational curve:

$$
\begin{array}{r}
F=\mathbb{P}_{\mathbb{P}^{1}}(\mathcal{E})=\mathbb{P}_{\mathbb{P}^{1}}\left(\mathcal{O}\left(a_{1}\right) \oplus \mathcal{O}\left(a_{2}\right) \oplus \cdots \oplus \mathcal{O}\left(a_{n-2}\right)\right) \\
\quad \text { with } \quad a_{1} \leq a_{2} \leq \cdots \leq a_{n-2} .
\end{array}
$$

Consider the section $C \subset F$ corresponding to the surjection $\mathcal{E} \longrightarrow \mathcal{O}_{\mathbb{P}^{1}}\left(a_{1}\right)$; since $L . C_{0}=1$, the restriction of $L$ to $F$ is a twist of $\xi_{\mathcal{E}}$ and so the intersection number $d=L . C$ is minimal among horizontal sections; in $N_{1}(X)$, since the contraction is elementary, we have $C \equiv d C_{0}$.

Consider the family $\mathcal{C}$ of deformations of $C$ inside $X$; by Remark 3.2 these deformations are contained in $F$, and so they cannot break: Suppose, by contradiction, that a deformation $\widetilde{C}$ of $C$ is reducible; let $\widetilde{C}=\sum_{1}^{k} C_{i}$ and let $f$ be a fiber of the bundle projection $p$. Since $\widetilde{C} . f=1$ exactly one of the $C_{i}$ must be horizontal, and the others contained in the fibers, but, by the minimality of L.C among horizontal sections, this is impossible, hence the family $\mathcal{C}$ is an unsplit family. By Proposition 2.3.5

$$
\begin{aligned}
\operatorname{dim} \operatorname{Locus}(\mathcal{C}, 0 \rightarrow \mathrm{c}) & \geq \operatorname{dim} X-\operatorname{dim} \operatorname{Locus}(\mathcal{C})+\operatorname{deg}_{-\mathrm{K}}(\mathcal{C})-1 \\
& \geq 2+d(n-4)-1 \geq n-3
\end{aligned}
$$


then, using Lemma 2.3.4 and Serre's inequality

$$
\begin{aligned}
\operatorname{dim}(\operatorname{Locus}(\mathcal{C}, 0 \rightarrow \mathrm{c}) \cap \mathrm{f}) & \geq \operatorname{dim} \operatorname{Locus}(\mathcal{C}, 0 \rightarrow \mathrm{c})+\operatorname{dim} \mathrm{f}-\operatorname{dim} \mathrm{F} \\
& \geq 2(n-3)-(n-2)=n-4,
\end{aligned}
$$

a contradiction, since $n \geq 5$.

We can thus give a complete (and effective) description of smooth fibers:

Proposition 5.1.2. Let $\varphi: X \rightarrow Z$ be a small elementary contraction of a smooth variety, supported by $K_{X}+(n-4) L$, and let $F$ be a smooth fiber of $\varphi$. Then $F$ is either $\mathbb{P}^{n-2}, \mathbb{Q}^{n-2}$ or $\mathbb{P}^{n-3}$.

Proof. Let $E$ be an irreducible component of the exceptional locus of $\varphi$ containing $F$; the fiber locus inequality (Proposition 2.3.6) combined with our hypothesis gives

$$
2 n-4 \geq \operatorname{dim} E+\operatorname{dim} F \geq n+(n-4)-1 .
$$

If $\operatorname{dim} F=n-2$ then $E=F$; the hypothesis of Theorem 4.1 are satisfied, the case of the $\mathbb{P}$-bundle over a rational curve is ruled out by Proposition 5.1.1.

If $\operatorname{dim} F=n-3$ then $F \simeq \mathbb{P}^{n-3}$ by $[\mathbf{A B W} 2,1.1]$.

From now on we will restrict our study to the case $n=5$; the general case will then follow by horizontal slicing (see 5.6).

5.2. Scheme theoretic fiber. Let $\varphi: Y \rightarrow W$ be a small contraction of a smooth fivefold; we choose a smooth fiber $F=\varphi^{-1}(w)$ and consider a local contraction $\varphi: X \rightarrow Z$ around $F$; by Proposition 5.1.2 we know the geometric structure of $F$, i.e., the set theoretic pre-image $\varphi^{-1}(w)$ equipped with the reduced scheme structure, which is one of the following: $\mathbb{P}^{3}, \mathbb{Q}^{3}$ or $\mathbb{P}^{2}$. The next step in understanding the map is the study of the conormal bundle of $F$ in $X$, defined as the quotient $\mathcal{I}_{F} / \mathcal{I}_{F}^{2}$, where $\mathcal{I}_{F}$ is the ideal of $F$ with the reduced structure. We first establish some cohomological conditions which the normal bundle has to satisfy.

Lemma 5.2.1 ([AW2, 1.2.2]). Let $\varphi: X \rightarrow Z$ be a Fano-Mori or crepant birational contraction, $z \in Z, F=\varphi^{-1}(z)$, and $F^{\prime}$ any subscheme whose support is contained in $F$. Then

$$
H^{r}\left(F^{\prime}, \mathcal{O}_{F^{\prime}}\right)=0 \quad \text { for } \quad r \geq \operatorname{dim} F .
$$

Corollary 5.2.2. Let $F$ be a s-dimensional fiber of a Fano-Mori or crepant contraction such that $s>1$ and $H^{s-1}\left(F, \mathcal{O}_{F}\right)=0$ and let $N^{*}$ be its conormal bundle. Then

$$
H^{s}\left(F, N^{*}\right)=0
$$


Proof. Let $\mathcal{J}$ be the ideal of $F$ in $X$ and consider the exact sequence

$$
0 \longrightarrow \mathcal{J} / \mathcal{J}^{2}=N^{*} \longrightarrow \mathcal{O}_{X} / \mathcal{J}^{2} \longrightarrow \mathcal{O}_{X} / \mathcal{J} \longrightarrow 0 .
$$

Our claim follows by the long exact sequence

$$
\ldots \longrightarrow H^{s-1}\left(F, \mathcal{O}_{F}\right) \longrightarrow H^{s}\left(F, N^{*}\right) \longrightarrow H^{s}\left(F, \mathcal{O}_{X} / \mathcal{J}^{2}\right) \longrightarrow \ldots
$$

and Lemma 5.2.1.

Another cohomological condition holds for normal bundles of fibers of dimension three.

Lemma 5.2.3. Let $F$ be a 3-dimensional smooth fiber of a small contraction of a smooth variety of dimension five, and let $N:=N_{F / X}$ be its normal bundle. Then $h^{0}(N)-h^{1}(N) \leq 0$.

Proof. By Proposition 2.3.6 in this case $F$ coincides with the irreducible component of the exceptional locus in which it is contained, and so $F$ cannot move in $X$; since the dimension of its deformation space is greater or equal than $h^{0}(N)-h^{1}(N)$, the lemma follows.

As a last preparatory step we compute the first chern class of the conormal bundles.

Lemma 5.2.4. Let $\varphi: X \rightarrow Z$ be a local small contraction of a smooth variety of dimension five around a smooth fiber $F$, and let $N^{*}:=N_{F / X}^{*}$ be its conormal bundle; the possibilities for the first chern class of $N^{*}$ are given in the following table, where $l(\varphi)$ denotes the length of $\varphi$ :

\begin{tabular}{|c|c|c|}
\hline$F$ & $l(\varphi)$ & $c_{1}\left(N^{*}\right)$ \\
\hline $\mathbb{P}^{3}$ & 1 & 3 \\
& 2 & 2 \\
$\mathbb{Q}^{3}$ & 1 & 2 \\
$\mathbb{P}^{2}$ & 1 & 2 \\
\hline
\end{tabular}

Proof. The result follows from Proposition 5.1.2 observing that, by Proposition 2.3.6,

$$
6 \geq 2 \operatorname{dim} E \geq 5+l(\varphi)-1 .
$$

We have $l(\varphi) \leq 2$, and equality holds if $l(\varphi)=2$; in this case by [ABW2, Lemma 1.1] we have $F \simeq \mathbb{P}^{3}$. Then we apply the adjunction formula. 


\subsection{Nefness of the conormal bundle.}

Definition 5.3.1. Let $\varphi: X \rightarrow Z$ be a local contraction of a smooth variety around a smooth fiber $F$ and let $L$ be a $\varphi$-ample line bundle; we say that $L$ is $\varphi$-spanned if the evaluation morphism $\varphi^{*} \varphi_{*} L \rightarrow L$ is surjective at every point of $F$.

We will now prove that the relative spannedness of the anticanonical bundle is a sufficient condition for the nefness of $N_{F / X}^{*}$.

Proposition 5.3.2. Let $\varphi: X \rightarrow Z$ be a local small contraction of a smooth variety of dimension five around a smooth fiber $F$; if $-K_{X}$ is $\varphi$-spanned then $N_{F / X}^{*}$ is nef.

Proof. We divide our proof into four cases, according to the possibilities given by Lemma 5.2.4.

Case 1. $F \simeq \mathbb{P}^{3}$ and $c_{1}\left(N^{*}\right)=3$.

Since $\left(-K_{X}\right)_{\mathbb{P}^{3}} \simeq \mathcal{O}_{\mathbb{P}^{3}}(1)$ and $-K_{X}$ is $\varphi$-spanned, $-K_{X}$ is also $\varphi$-very ample; this implies that, for $l$ a line in $F, N_{l / X}^{*}(1)$ is spanned by global sections ([AW2, Lemma 1.3.5]).

Moreover we can find two sections $H_{1}, H_{2} \in\left|-K_{X}\right|$ such that $l=F \cap$ $H_{1} \cap H_{2}$, so that by formula $(2.2 .3)$

$$
\left(N_{F / X}^{*}\right)_{\mid l} \oplus\left(K_{X}\right)_{\mid l}^{\oplus 2}=N_{l / X}^{*},
$$

allowing for $N_{F / X}^{*}$ the following splitting types on lines: $(4,-1),(3,0)$ and $(2,1)$.

If the general splitting of $N_{F / X}^{*}$ were $(4,-1)$, then $N_{F / X}^{*} \simeq \mathcal{O}_{\mathbb{P}^{3}}(4) \oplus$ $\mathcal{O}_{\mathbb{P}^{3}}(-1)$, but, in this case we would have $h^{0}\left(N_{F / X}\right)-h^{1}\left(N_{F / X}\right)>0$ and this is impossible by Lemma 5.2.3.

The restriction of $N_{F / X}^{*}$ to the generic line is thus nef, hence spanned; applying Lemma 2.2.4 twice we have that $N_{F / X}^{*}$ is generically spanned on $F$, hence nef.

Case 2. $\quad F \simeq \mathbb{P}^{3}$ and $c_{1}\left(N^{*}\right)=2$.

In this case we have $\left(-K_{X}\right)_{F} \simeq \mathcal{O}_{\mathbb{P}^{3}}(2)$.

Claim. Either there exists a line bundle $\mathcal{L}$ on $X$ such that $\mathcal{L}_{F} \simeq \mathcal{O}_{\mathbb{P}^{3}}(1)$, or $N^{*}=\mathcal{O}_{\mathbb{P}^{3}}(1)^{\oplus 2}$.

Proof of the claim. Let $A$ be a very ample divisor on $Z$ and $H=\varphi^{*} A$; for $m$ large enough the linear system $\left|m H+K_{X}\right|$ is nonempty, so there exists an effective divisor $D^{\prime}$ such that $D^{\prime} . l<0$ for $l$ a line in $F$; in particular there exists an irreducible reduced divisor $D$ s.t. $D . l<0$.

If $D . l$ is odd, then a combination of $D$ and $-K_{X}$ gives us a line bundle $\mathcal{L}$ such that $\mathcal{L}_{F} \simeq \mathcal{O}_{\mathbb{P} 3}(1)$. 
So suppose that $D . l=-2 t$ and consider $\beta: \hat{X} \rightarrow X$, the blow up $X$ along $F$ with exceptional divisor $\hat{F}=\mathbb{P}\left(N_{F / X}^{*}\right)$ and the strict transform of $D, \bar{D}=\beta^{*} D-2 t E$.

Let $l$ be a line in $\mathbb{P}^{3}$; an easy computation shows that the restriction of $\bar{D}$ to $\mathbb{P}_{l}\left(N_{F / X}^{*}\right) \simeq \mathbb{F}_{e}$ has a component corresponding to $t$-times the fundamental section (which has to be contained in $\bar{D}$ since has negative intersection with it); hence there exist divisors $D_{1}, D_{2}$ in $\left|-t E+p^{*} \mathcal{O}_{\mathbb{P}^{3}}(a)\right|$, $\left|-t E+p^{*} \mathcal{O}_{\mathbb{P}^{3}}(b)\right|$, with $a+b=-2$ (we can possibly have $\left.D_{1}=D_{2}\right)$.

Another straightforward computation on the ruled surfaces $\mathbb{P}_{l}\left(N_{F / X}^{*}\right)$ shows that we have $e=b-a$ for every line. It follows that the splitting type of $N^{*}$ is constant on lines, hence $N^{*}$ is decomposable and so, by Lemma 5.2.3 the only possibility is $N^{*}=\mathcal{O}_{\mathbb{P}^{3}}(1)^{\oplus 2}$.

So we can suppose that there exists a line bundle $\mathcal{L}$ on $X$ such that $\mathcal{L}_{F} \simeq \mathcal{O}_{\mathbb{P}^{3}}(1) ;$ up to twist $\mathcal{L}$ with $\varphi^{*} A$ with $A$ very ample on $Z$ we can assume that $\mathcal{L}$ is ample. Let $X^{\prime}$ be a generic divisor in $|\mathcal{L}|$ and $F^{\prime}=F \cap X^{\prime}$; by Lemma 2.2.1 $X^{\prime}$ is smooth and $K_{X^{\prime}}+\mathcal{L}_{X^{\prime}}$ is a good supporting divisor of a small contraction of a smooth fourfold. By $[\mathbf{K a 2}] F^{\prime} \simeq \mathbb{P}^{2}$ and $N_{F^{\prime}} / X^{\prime} \simeq$ $\oplus^{2} \mathcal{O}_{\mathbb{P}^{2}}(-1) ;$ since by formula $(2.2 .3)$

$$
\left(N_{F / X}\right)_{\mid F^{\prime}}=N_{F^{\prime} / X^{\prime}}
$$

we have that $N_{F / X} \simeq \oplus^{2} \mathcal{O}_{\mathbb{P}^{3}}(-1)$.

Case 3. $E \simeq \mathbb{Q}^{3}$.

Let $X^{\prime}$ be a general divisor in $\left|-K_{X}\right|, F^{\prime}=F \cap X^{\prime} \simeq \mathbb{Q}^{2}$ and $N:=N_{F^{\prime}} / X^{\prime}$. By [AW2, Lemma 2.10.1], if the generic splitting type of $N$ on conics is not $(-2,-2)$ then there exist a zero dimensional subscheme $T \subset F^{\prime}$ and an exact sequence:

$$
0 \longrightarrow \mathcal{O}_{\mathbb{Q}^{2}}\left(a_{1}, a_{2}\right) \longrightarrow N \longrightarrow \mathcal{I}_{T} \otimes \mathcal{O}_{\mathbb{Q}^{2}}\left(b_{1}, b_{2}\right) \longrightarrow 0
$$

Twisting our sequence by $\mathcal{O}_{\mathbb{Q}^{2}}(-2)$ we have:

$$
0 \longrightarrow \mathcal{O}_{\mathbb{Q}^{2}}\left(a_{1}-2, a_{2}-2\right) \longrightarrow N(-2) \longrightarrow \mathcal{I}_{T} \otimes \mathcal{O}_{\mathbb{Q}^{2}}\left(b_{1}-2, b_{2}-2\right) \longrightarrow 0 .
$$

By Serre duality and Lemma 5.2.1 we have

$$
H^{0}\left(\mathbb{Q}^{2}, N(-2)\right)=H^{0}\left(\mathbb{Q}^{2}, K_{\mathbb{Q}^{2}} \otimes N\right) \simeq H^{2}\left(\mathbb{Q}^{2}, N^{*}\right)=0 ;
$$

since $H^{0}\left(\mathbb{Q}^{2}, N(-2)\right)=0$, by the long cohomology exact sequence, we have $H^{0}\left(\mathbb{Q}^{2}, \mathcal{O}\left(a_{1}-2, a_{2}-2\right)\right)=0$, yielding $a_{1}, a_{2} \leq 1$ (and $b_{1}, b_{2} \geq-3$ ).

Observe that, since $-K_{X}$ is $\varphi$-spanned and $-K_{X \mid F^{\prime}} \simeq \mathcal{O}_{\mathbb{Q}^{2}}(1)$, either $-K_{X}$ is $\varphi$-very ample or the image of the vector space $H^{0}\left(X^{\prime},-K_{X \mid X^{\prime}}\right)$ in the space $H^{0}\left(\mathbb{Q}^{2}, \mathcal{O}_{\mathbb{Q}^{2}}(1)\right)$ corresponds to a double cover $\pi: \mathbb{Q}^{2} \rightarrow \mathbb{P}^{2}$.

In both cases it is possible to find a section $X^{\prime \prime} \in\left|\left(-K_{X}\right)_{\mid X^{\prime}}\right|$ such that $F^{\prime \prime}=X^{\prime} \cap X^{\prime \prime}$ is a smooth conic passing through two points of $T$; the 
splitting type of $N$ on such a conic would then be $(b-4, a+4)$ and $X^{\prime \prime}$ would contain an exceptional curve with normal bundle $(b-4, a+4)$; by $[\mathbf{N a}]$ this implies that either $(a, b)=(-1,-3),(-2,-2)$ or $T$ consists of a single point, but in this case we would have $h^{0}(N)-h^{1}(N)>0$, and this is impossible by Lemma 5.2.3.

It follows that on the generic conic $N$ has splitting type $(-1,-3)$ or $(-2,-2)$, hence $N^{*}$ is spanned on the generic conic and we can apply Lemma 2.2.4.

Case 4. $\quad E \simeq \mathbb{P}^{2}$.

Let $X^{\prime} \in\left|-K_{X}\right|$ be any divisor, and $l=F \cap X^{\prime}$; the first cohomology group of the conormal bundle of $l$ in $X^{\prime}$ vanishes [AW2, Proposition 5.6.1], so, since by formula (2.2.3)

$$
\left(N_{F / X}^{*}\right)_{\mid l}=N_{l / X^{\prime}}^{*}
$$

the possible splitting types of $N^{*}$ are $(1,-3),(2,0)$, and $(1,1)$; if the general splitting type is different from $(1,-3)$ then by Lemma $2.2 .4 N^{*}$ is nef and we are done. If the general splitting of $N^{*}$ is $(1,-3)$ then $N^{*} \simeq \mathcal{O}(1) \oplus \mathcal{O}(-3)$, but this is impossible by Lemma 5.2.3.

Remark 5.3.3. By results of Mella $[\mathrm{Me}]$ we know that, for the contractions that we are studying, the anticanonical bundle has finite base locus on $F$; we believe that, in the set up of Proposition 5.3.2 it should be true that the anticanonical bundle is always $\varphi$-spanned.

5.4. Description of the conormal bundle. The nefness of the conormal bundles implies that they are Fano bundles; this fact allows us to give a description of them:

Proposition 5.4.1. Let $\varphi: X \rightarrow Z$ be a small contraction of a smooth variety of dimension 5 such that $-K_{X}$ is $\varphi$-spanned, and $F$ a smooth fiber of $\varphi$. Then $\left(F, N_{F / X}\right)$ is one of the following:

\begin{tabular}{|r|c|}
\hline$F$ & $N_{F / X}$ \\
\hline $\mathbb{P}^{3}$ & $\mathcal{O}_{\mathbb{P}^{3}}(-1) \oplus \mathcal{O}_{\mathbb{P}^{3}}(-1)$ \\
& $\mathcal{O}_{\mathbb{P}^{3}}(-2) \oplus \mathcal{O}_{\mathbb{P}^{3}}(-1)$ \\
$\mathbb{Q}^{3}$ & $\mathcal{O}_{\mathbb{Q}^{3}}(-1) \oplus \mathcal{O}_{\mathbb{Q}^{3}}(-1)$ \\
$\mathbb{P}^{2}$ & $\mathcal{O}_{\mathbb{P}^{2}} \oplus \mathcal{O}_{\mathbb{P}^{2}}(-1)^{\oplus 2}$ \\
\hline
\end{tabular}

Proof. The anticanonical bundle of the projectivization $\mathbb{P}\left(N^{*}\right)$ is given by the formula

$$
-K_{\mathbb{P}\left(N^{*}\right)}=-p^{*}\left(K_{F}+c_{1}\left(N^{*}\right)\right)+2 \xi_{N^{*}},
$$

where $p: \mathbb{P}\left(N^{*}\right) \rightarrow F$ is the bundle projection; so it is ample, being the sum of two nef divisors, the first of which vanishes only on the fibers of $p$, where 
the second is positive. It follows that $N^{*}$ is a Fano bundle over $\mathbb{P}^{3}, \mathbb{Q}^{3}$ or $\mathbb{P}^{2}$ i.e., its projectivization is a Fano manifold. In the rest of the proof we will use the following:

Lemma 5.4.2 ([AW2, 5.3 and 5.3.1]). Let $\varphi: X \rightarrow Z$ be a good or crepant contraction of a smooth variety and let $F$ be a smooth fiber of $\varphi$. Consider the blow-up of $X$ along $F, \beta: \hat{X} \rightarrow X$ and denote by $\hat{F}$ the exceptional divisor.

If the conormal bundle $N_{F}^{*}$ is nef then the line bundle $\mathcal{O}_{\hat{X} / X}(1)=-\hat{F}$ is $\varphi \circ \beta$-nef, some positive multiple $\mathcal{O}_{\hat{X} / X}(k)=-\hat{k} F$ is $\varphi \circ \beta$-spanned and it defines a good contraction $\hat{\varphi}$ over $W$. The restriction of $\hat{\varphi}$ to $\hat{F}$ is induced by the evaluation map $\bigoplus H^{0}\left(S^{k}\left(N^{*}\right)\right) \rightarrow \bigoplus S^{k}\left(N^{*}\right)$.

Corollary 5.4.3. Suppose that $N_{F}^{*}$ is a nonample Fano-bundle, that $\hat{\varphi}_{\hat{F}}$ has one dimensional fibers and that either $\hat{\varphi}_{\hat{F}}$ is birational or has length $\geq 2$; then $\varphi$ cannot be small.

Proof. The restriction of $\hat{\varphi}$ to $\hat{F}$ is an extremal contraction of $\hat{F}$ and we have $l(\hat{\varphi}) \geq l\left(\hat{f}_{\mid \hat{F}}\right)$. Proposition 2.3.6 yields

$$
\operatorname{dim} E(\hat{\varphi}) \geq n+l(\varphi)-2,
$$

so that $E(\hat{\varphi})=\hat{X}$ if $\hat{f}_{\mid \hat{F}}$ has length $\geq 2$, and $E(\hat{\varphi})$ is a divisor if $\varphi_{\hat{F}}$ is birational; both the situations are impossible since, outside $\hat{F}$, the exceptional loci of $\varphi$ and $\hat{\varphi}$ coincide.

Now we resume the proof of the proposition, starting with the case of a 3-dimensional fiber; by the classification of Fano bundles [SW1] we have the following possibilities:

\begin{tabular}{|c|c|c|}
\hline $\mathbb{P}^{3}, c_{1}\left(N^{*}\right)=3$ & $\mathbb{P}^{3}, c_{1}\left(N^{*}\right)=2$ & $\mathbb{Q}^{3}, c_{1}\left(N^{*}\right)=2$ \\
\hline $\mathcal{O}_{\mathbb{P}^{3}}(2) \oplus \mathcal{O}_{\mathbb{P}^{3}}(1)$ & $\mathcal{O}_{\mathbb{P}^{3}}(1) \oplus \mathcal{O}_{\mathbb{P}^{3}}(1)$ & $\mathcal{O}_{\mathbb{Q}^{3}}(1) \oplus \mathcal{O}_{\mathbb{Q}^{3}}(1)$ \\
$\mathcal{O}_{\mathbb{P}^{3}} \oplus \mathcal{O}_{\mathbb{P}^{3}}(3)$ & $\mathcal{O}_{\mathbb{P}^{3}} \oplus \mathcal{O}_{\mathbb{P}^{3}}(2)$ & $\pi^{*} \mathcal{N}(1)$ \\
& $\mathcal{N}(1)$ & \\
\hline
\end{tabular}

where $\mathcal{N}$ is a null correlation bundle over $\mathbb{P}^{3}$ (see $[\mathbf{S W 1}$, Section 2]) and $\pi: \mathbb{Q}^{3} \rightarrow \mathbb{P}^{3}$ is a double cover.

The bundles with a trivial summand have the property that $h^{0}(N)-$ $h^{1}(N) \geq 0$, so they have to be excluded by Lemma 5.2 .3 , while the null correlation bundle over $\mathbb{P}^{3}$ and its pullback to $\mathbb{Q}^{3}$ are ruled out by Corollary 5.4.3.

Now we can study the case in which $F$ is two-dimensional; the classification of Fano bundles on $\mathbb{P}^{2}[\mathbf{S W 2}]$ gives us the following possibilities: 


\begin{tabular}{|c|c|c|}
\hline Description of the bundle & $\hat{\varphi}_{\mid \hat{F}}$ & dim fibers $\hat{\varphi}_{\mid \hat{F}}$ \\
\hline $\mathcal{O}(1) \oplus \mathcal{O}(1) \oplus \mathcal{O}$ & Small & 2 \\
$\mathcal{O}(2) \oplus \mathcal{O} \oplus \mathcal{O}$ & Divisorial & 2 \\
$T \mathbb{P}^{2}(-1) \oplus \mathcal{O}(1)$ & Divisorial & 1 \\
$\mathcal{O} \oplus \mathcal{E}_{2}$ with $\mathcal{E}_{2}$ in & Divisorial & 1 \\
$0 \rightarrow \mathcal{O} \rightarrow \mathcal{E}_{2}(-1) \rightarrow J_{x} \rightarrow 0$ & & \\
$0 \rightarrow \mathcal{O}(-1)^{\oplus 2} \rightarrow \mathcal{O}^{3} \rightarrow \mathcal{E} \rightarrow 0$ & Divisorial & 1 \\
$0 \rightarrow \mathcal{O} \rightarrow \mathcal{E}_{3} \rightarrow \mathcal{E}_{2}$ & Divisorial & 1 \\
$0 \rightarrow \mathcal{O}(-2) \rightarrow \mathcal{O}^{\oplus 3} \rightarrow \mathcal{E} \rightarrow 0$ & Fiber type & 1 \\
\hline
\end{tabular}

All the bundles with $\hat{\varphi}$ divisorial with one-dimensional fibers are ruled out by Corollary 5.4.3; the bundle $\mathcal{O}(2) \oplus \mathcal{O}^{\oplus 2}$ has also to be excluded, because for this bundle $h^{0}(N)-h^{1}(N) \geq 2$ and $F$ would move too much.

The last bundle is excluded observing that $H^{1}\left(N^{*}\right)=0$ and $H^{0}\left(S^{k} N^{*}\right)=$ $S^{k} H^{0}\left(N^{*}\right)$, so that by [AW2, Proposition 2.4] the contraction of $F$ should be to a smooth 4-dimensional point.

Note that in every case the conormal bundle $N_{F / X}^{*}$ is spanned by global sections; using [AW2, Proposition 5.4] we can conclude that the schemetheoretic structure of $F$, i.e., the closed subscheme defined by the ideal $\mathcal{I}_{\widetilde{F}} \varphi^{-1}\left(m_{z}\right) \dot{\mathcal{O}}_{X}$, coincides with the geometric structure of $F$.

Moreover, the formal neighbourhood of $F$ in $X$ is determined uniquely and it is the same as the formal neighbourhood of the zero section of the total space of the bundle $N^{*}$, as follows from a criterion of Mori [Mo2, 3.33]:

Proposition 5.4.4. Suppose that $F$ is a smooth fiber of a Fano-Mori or crepant contraction $\varphi: X \rightarrow Z$, and assume that the conormal bundle $N_{F / X}^{*}$ is nef. If $H^{1}\left(F, T_{F} \otimes S^{i}\left(N^{*}\right)\right)=H^{1}\left(F, N \otimes S^{i}\left(N^{*}\right)\right)=0$ for $i \geq 1$ then the formal neighbourhood of $F$ in $X$ is determined uniquely and it is the same as the formal neighbourhood of the zero section in the total space of the bundle $N^{*}$.

5.5. Description of the flip. In the case of a three dimensional fiber consider again $\beta: \hat{X} \rightarrow X$, the blow up of $X$ along $F$, and denote by $\hat{F}$ the exceptional divisor; since in our case $N_{F}^{*}$ is ample, the line bundle $-\hat{F}$ on $\hat{X}$ is $(\varphi \circ \beta)$ ample and hence $-K_{\hat{X}}$ is $(\varphi \circ \beta)$ ample.

Since $\rho(\hat{X} / Z)=2$ there is a Fano-Mori contraction $\phi: \hat{X} \rightarrow X^{+}$over $Z$, different from $\varphi$; easy computations show that the line bundle $K_{\hat{X}}-$ $(l(\varphi)+1) \hat{F}$ is $\varphi \circ \beta$ nef and is a good supporting divisor for the contraction $\phi: \hat{X} \rightarrow X^{+}$; if $N_{F}^{*}$ is $\mathcal{O}(1) \oplus \mathcal{O}(1)$ then $\phi$ is a divisorial contraction with 
fibers isomorphic to $\mathbb{P}^{3}$ and $\mathbb{Q}^{3}$ respectively, and the flip is given by the following diagram:

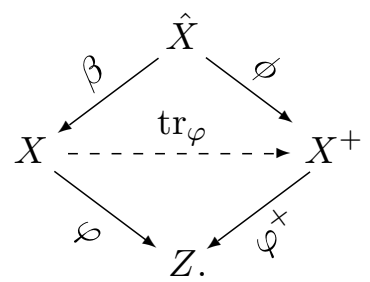

Otherwise, if $\left(F, N_{F}^{*}\right) \simeq\left(\mathbb{P}^{3}, \mathcal{O}_{\mathbb{P}}(2) \oplus \mathcal{O}_{\mathbb{P}}(1)\right)$ then $\phi$ is a small contraction of a $\mathbb{P}^{3}$ with normal bundle $\mathcal{O}_{\mathbb{P}}(-1) \oplus \mathcal{O}_{\mathbb{P}}(-1)$, which falls in the previous case, so that the flip is given by the following diagram:

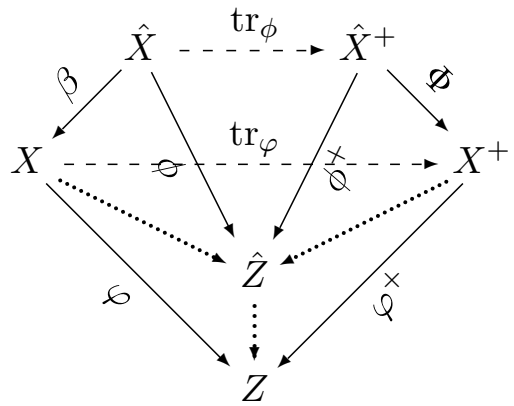

where $\Phi$ is the contraction of a $\mathbb{P}^{4}$ with normal bundle $\mathcal{O}_{\mathbb{P}}(-2)$ to a point.

In the case of 2-dimensional fibers we blow up $X$ along $E(\varphi)$; the exceptional divisor of the blow up is a fiber product of a $\mathbb{P}^{2}$ and a $\mathbb{P}^{1}$-bundle over $B$; we can contract $\hat{X}$ to a smooth variety by contracting the exceptional divisor to the $\mathbb{P}^{1}$-bundle over $B$ (see $[\mathbf{A B W} \mathbf{1}]$ ).

5.6. The case $\boldsymbol{n}>\mathbf{5}$. Take $n-5$ generic sections $H_{1}, \ldots H_{n-5}$ in $|L|$ and consider the variety $X^{\prime}=X \cap\left(\cap_{i}^{n-5} H_{i}\right)$; by Lemma 2.2.1 $X^{\prime}$ is smooth and $K_{X}^{\prime}+L_{X^{\prime}}$ is a good supporting divisor of a small contraction of $X^{\prime}$; since $F$ is a projective space or a three dimensional quadric and $L_{F} \simeq \mathcal{O}(1)$, we have that $F^{\prime}=F \cap\left(\cap_{i}^{n-5} H_{i}\right)$ is smooth and is again a projective space or a quadric. Its conormal bundle is the restriction of the conormal bundle of $F$; moreover by Lemma 2.2.4 the bundle $N_{F / X}^{*}$ is nef. Note that, if $n>5$ then $l(\varphi)=n-4$, so we cannot obtain the pair $\left(\mathbb{P}^{3}, \mathcal{O}_{\mathbb{P}^{3}}(1) \oplus \mathcal{O}_{\mathbb{P}^{3}}(1)\right)$ as $\left(F^{\prime}, N_{F^{\prime} / X^{\prime}}^{*}\right)$; the flip of $\varphi$ is constructed exactly as in the case $n=5$.

\section{Examples.}

Example 6.1 (small contractions with fibers $\left.\simeq \mathbb{P}^{3}, \mathbb{Q}^{3}\right)$. Let $\left(F^{\prime}, \mathcal{E}\right)$ be a pair consisting of a smooth Fano variety $F^{\prime}$ and a numerically effective vector bundle $\mathcal{E}$ such that $-K_{F^{\prime}}-\operatorname{det} \mathcal{E}$ is ample. 
Consider the projectivization $X:=\mathbb{P}(\mathcal{E} \oplus \mathcal{O})$ and the section of the projective bundle $\pi: X \rightarrow F^{\prime}$ determined by the surjection $\mathcal{E} \oplus \mathcal{O} \longrightarrow \mathcal{O} \longrightarrow 0$; denote this section by $F$; it is easy to check that $N_{F / X}^{*}=\mathcal{E}$.

Let $\xi$ denote the tautological line bundle over $X$; since $\xi$ is nef and $\xi-K_{\mathbb{P}(\mathcal{E} \oplus \mathcal{O})}$ is ample, by the Contraction theorem it follows that the linear system $|m \xi|$ is base point free for $m>>0$ and defines a Fano-Mori contraction $\varphi: X \rightarrow W$ with $W=\mathbb{P}\left(\oplus_{m \geq 0} H^{0}\left(S^{m}(\mathcal{E} \oplus \mathcal{O})\right)\right)$.

If $\mathcal{E}$ is ample the map $\varphi$ is just the contraction of $F$ to a point; considering as $\left(F^{\prime}, \mathcal{E}\right)$ the pairs

\begin{tabular}{|c|c|}
\hline$F^{\prime}$ & $\mathcal{E}$ \\
\hline $\mathbb{P}^{3}$ & $\mathcal{O}_{\mathbb{P}^{3}}(1) \oplus \mathcal{O}_{\mathbb{P}^{3}}(1)$ \\
& $\mathcal{O}_{\mathbb{P}^{3}}(2) \oplus \mathcal{O}_{\mathbb{P}^{3}}(1)$ \\
$\mathbb{Q}^{3}$ & $\mathcal{O}_{\mathbb{Q}^{3}}(1) \oplus \mathcal{O}_{\mathbb{Q}^{3}}(1)$ \\
\hline
\end{tabular}

we construct examples of small contractions with smooth fibers of dimension three.

Example 6.2 (a small contraction with fibers $\simeq \mathbb{P}^{2}$ ). Let $F^{\prime}=\mathbb{P}^{2}$ and $\mathcal{E}=$ $\mathcal{O}_{\mathbb{P}^{2}}(1) \oplus \mathcal{O}_{\mathbb{P}^{2}}(1)$; the construction of the previous example gives us a small contraction of a smooth fourfold $f: X^{\prime} \rightarrow W^{\prime}$ which contracts a projective plane to a point.

Let $B$ be a smooth curve; the map $\varphi:=f \times 1_{B}: X^{\prime} \times B \rightarrow W^{\prime} \times B$ is a Fano-Mori contraction of a smooth variety of dimension five with fibers $\simeq \mathbb{P}^{2}$.

Example 6.3 (small contractions with singular quadrics as fibers). (The following construction was inspired by [AW3], Section 4, and [Ka2].)

Let $S \subset \mathbb{C}^{6}$ be the hypersurface defined by the equation

$$
z_{1}^{2}+z_{2}^{2}+z_{3}^{2}+z_{4}^{2}+z_{5}^{3}=0
$$

$C \subset S$ the curve $z_{1}=z_{2}=\cdots=z_{5}=0$, and $Z \subset S$ the threefold $\sum_{i=1}^{4} z_{i}^{2}=z_{5}=z_{6}=0$.

Consider the blow-up of $S$ along $C, \beta: Y \rightarrow S$; every fiber of $\beta$ is a three dimensional cone with a singular point.

The strict transform $\bar{Z}$ of $Z$ is smooth and meets $F_{0}=\beta^{-1}(0,0,0,0,0,0)$ along a smooth two-dimensional quadric; consider now $\sigma: X \rightarrow Y$, the blow up of $Y$ along $\bar{Z}$. The variety $X$ obtained in this way is smooth, its anticanonical divisor $-K_{X}$ is $(\sigma \circ \beta)$-ample, and $\rho(X / S)=2$, so there exists an extremal ray on $X$ not contracted by $\sigma$; this ray is spanned by the class of a line in $F$, the strict transform of $F_{0}$; the contraction of this ray contracts $F$ to a point and it is a small contraction.

In the same way we can construct a small contraction with a fiber isomorphic to a singular three dimensional quadric with $\operatorname{dim} \operatorname{Sing}(\mathbb{Q})=1$, taking 
the hypersurface

$$
z_{1}^{2}+z_{2}^{2}+z_{3}^{2}+z_{4}^{3}+z_{5}^{3}+z_{4}^{2} z_{6}+z_{5}^{2} z_{6}=0
$$

as $S$, the curve $z_{1}=z_{2}=\cdots=z_{5}=0$ as $C$ and the threefold $z_{1}^{2}+z_{2}^{2}+z_{3}^{2}+$ $z_{4}^{3}=z_{5}=z_{6}=0$ as $Z$.

Example 6.4 (a double covering). Consider a small contraction $\psi: Y \rightarrow$ $Z$ of a smooth fivefold which contracts a $\mathbb{P}^{3}$ with normal bundle $\mathcal{O}_{\mathbb{P}^{3}}(-1)^{\oplus 2}$ to a point (cf. Example 6.1); we have seen that such a contraction is supported by $K_{X}+2 L$, with $L$ a $\psi$-ample line bundle; in this example we can take $L=\pi^{*} \mathcal{O}_{\mathbb{P}^{3}}(1)$.

Take a smooth divisor $B \in|2 L|$ and construct a double covering $p: X \rightarrow$ $Y$ branched along $B$; the variety $X$ is smooth and $-K_{X}$ is $(\psi \circ \pi)$-ample. Let $\varphi: X \rightarrow W$ be the connected part of the Stein factorization of $(\psi \circ \pi)$; then $\varphi$ is a Fano-Mori contraction which fits in a commutative diagram

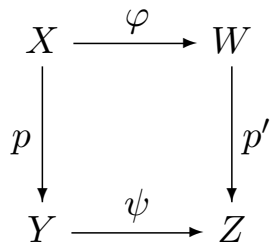

where $p^{\prime}: W \rightarrow Z$ is a double covering branched along $\psi(B)$.

The contraction $\varphi$ is a small contraction which contracts a smooth-three dimensional quadric to a point.

Example 6.5 (toric examples).

1) Let $N$ be a lattice of rank five and $\Delta^{\prime}$ the cone generated by a base $v_{1}, v_{2}, v_{3}, w_{1}, w_{2}$ of $N$ and by $v_{4}=w_{1}+w_{2}-v_{1}-v_{2}-v_{3}$; consider the subdivision $\Delta$ of $\Delta^{\prime}$ obtained considering the four cones

$$
\left\langle v_{1}, \ldots, \hat{v}_{i}, \ldots, v_{4}, w_{1}, w_{2}\right\rangle \text {. }
$$

The induced toric morphism $\varphi: X(\Delta) \rightarrow X\left(\Delta^{\prime}\right)$ is the contraction of $V\left(\left\langle w_{1}, w_{2}\right\rangle\right) \simeq \mathbb{P}^{3}$ with normal bundle $\mathcal{O}_{\mathbb{P}^{3}}(-1)^{\oplus 2}$ to a point. The flip of $\varphi$ is given by the following diagram:

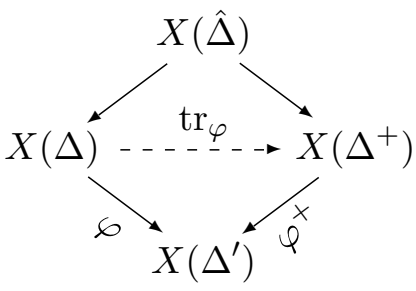


where $\hat{\Delta}$ is the subdivision of $\Delta$ obtained introducing the vector $u=$ $w_{1}+w_{2}$ and the cones $\left\langle v_{1}, \ldots \hat{v}_{i}, \ldots, v_{4}, w_{j}\right\rangle$, and $\Delta^{+}$is the subdivision of $\Delta^{\prime}$ obtained considering the two cones $\left\langle v_{1}, v_{2}, v_{3}, v_{4}, w_{1}\right\rangle$ and $\left\langle v_{1}, v_{2}, v_{3}, v_{4}, w_{2}\right\rangle$.

2) Again let $N$ be a lattice of rank five and $\Delta^{\prime}$ the cone generated by a base $v_{1}, v_{2}, v_{3}, w_{1}, w_{2}$ of $N$ and by $v_{4}=w_{1}+2 w_{2}-v_{1}-v_{2}-v_{3}$; consider the subdivision $\Delta$ of $\Delta^{\prime}$ obtained considering the four cones

$$
\left\langle v_{1}, \ldots, \hat{v}_{i}, \ldots, v_{4}, w_{1}, w_{2}\right\rangle \text {. }
$$

The induced toric morphism $\varphi: X(\Delta) \rightarrow X\left(\Delta^{\prime}\right)$ is the contraction of $V\left(\left\langle w_{1}, w_{2}\right\rangle\right) \simeq \mathbb{P}^{3}$, with normal bundle $\mathcal{O}_{\mathbb{P}^{3}}(-1) \oplus \mathcal{O}_{\mathbb{P}^{3}}(-2)$ to a point, and the flip is given by the following diagram:

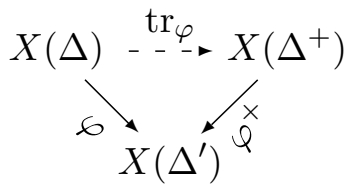

where $\Delta^{+}$is the subdivision of $\Delta^{\prime}$ obtained considering the two cones $\left\langle v_{1}, v_{2}, v_{3}, v_{4}, w_{1}\right\rangle$ and $\left\langle v_{1}, v_{2}, v_{3}, v_{4}, w_{2}\right\rangle$ (see [Re, Theorem 3.4]).

Note that, introducing in $\Delta$ the vectors $u=w_{1}+w_{2}$ and $v=$ $w_{1}+2 w_{2}$ and the appropriate cones, we can recover from the toric point of view the construction in 5.5.

Acknowledgements. This work grew out of a part of the author's Ph.D. thesis $[\mathbf{O c}]$ at the Department of Mathematics of the University of Trento: I would like to thank Marco Andreatta who proposed me the problem treated in this paper and helped me with precious suggestions.

I'm also indebted to Andrew Sommese, who gave me the idea of using the Albanese map in the Proof of Theorem 4.1 and to Jaroslaw Wiśniewski for many valuable comments and discussions.

\section{References}

[ABW1] M. Andreatta, E. Ballico and J.A. Wiśniewski, Projective manifolds containing large linear subspaces, in 'Classification of Irregular Varieties (Trento 1990)', Lecture Notes in Math., 1515, Springer Verlag, 1992, 1-11, MR 93i:14006, Zbl 0787.14034.

[ABW2] _ Two theorems on elementary contractions, Math. Ann., 297 (1993), 191-198, MR 94j:14020, Zbl 0789.14011.

[AO1] M. Andreatta and G. Occhetta, Special rays in the Mori cone of a projective variety, to appear in Nagoya Math. J., math.AG/0010101.

[AW1] M. Andreatta and J.A. Wiśniewski, A note on nonvanishing and applications, Duke Math. J., 72 (1993), 739-755, MR 95c:14007, Zbl 0853.14003. 

, On contractions of smooth varieties, J. Alg. Geom., 7 (1998), 253-312, MR 99f:14021a, Zbl 0966.14012.

[AW3] Contractions of smooth varieties II: Computations and applications, Boll. Unione Mat. Ital. Sez. B Artic. Ric. Mat. (8), 1 (1998), 343-360, MR 99f:14021b.

[BS] M.C. Beltrametti and A.J. Sommese, The Adjunction Theory of Complex Projective Varieties, Exp. Math., 16, de Gruyter, Berlin, 1995, MR 96f:14004, Zbl 0845.14003.

[Fu] T. Fujita, Remarks on quasi-polarized varieties, Nagoya Math. J., 115 (1989), 105-123, MR 90i:14045, Zbl 0699.14002.

[Io] P. Ionescu, Generalized adjunction and applications, Math. Proc. Camb. Phil. Soc., 99 (1986), 457-472, MR 87e:14031, Zbl 0619.14004.

[Ka1] Y. Kawamata, The cone of curves of algebraic varieties, Ann. of Math., 119 (1984), 603-633, MR 86c:14013c, Zbl 0544.14009.

[Ka2] - Small contractions of four dimensional algebraic manifolds, Math. Ann., 284 (1989), 595-600, MR 91e:14039, Zbl 0661.14009.

[KaMaMa] Y. Kawamata, K. Matsuda and K. Matsuki, Introduction to the minimal model program, in 'Algebraic Geometry, Sendai', Adv. Studies in Pure Math., 10, Kinokuniya-North-Holland, 1987, 283-360, MR 89e:14015, Zbl 0672.14006.

[Ke] S. Kebekus, Families of singular rational curves, J. Alg. Geom., 11 (2002), 245-256.

[Ko] J. Kollár, Rational Curves on Algebraic Varieties, Ergebnisse der Math., 32, Springer Verlag, Berlin, Heidelberg, New York, Tokio, 1996, MR 98c:14001, Zbl 0877.14012.

[KoMiMo] J. Kollár, Y. Miyaoka and S. Mori, Rational connectedness and boundedness of Fano manifolds, J. Diff. Geom., 36 (1992), 765-779, MR 94g:14021, Zbl 0759.14032.

[Me] M. Mella, On del Pezzo fibrations, Ann. Scuola Norm. Sup. Pisa Cl. Sci., 28 (1999), 615-639, MR 2001d:14009, Zbl 0981.14005.

[Mo1] S. Mori, Projective manifolds with ample tangent bundle, Ann. Math., 110 (1979), 595-606, MR 81j:14010, Zbl 0423.14006.

[Mo2] Threefolds whose canonical bundles are not numerically effective, Ann. Math., 116 (1982), 133-176, MR 84e:14032, Zbl 0557.14021.

[Na] N. Nakayama, On smooth exceptional curves in threefolds, J. Fac. Sci. Univ. Tokyo Sect. IA Math., 37 (1990), 511-525, MR 92e:14035, Zbl 0739.14027.

[Oc] G. Occhetta, Extremal Rays of Smooth Projective Varieties, UTM PhDTS 27, University of Trento, 1999.

[Re] M. Reid, Decomposition of toric morphisms, in 'Arithmetic and Geometry', Vol. II, Progr. Math., 36, Birkhäuser Boston, Boston, MA, 1983, 395-418, MR 85e:14071, Zbl 0571.14020.

[SW1] M. Szurek and J.A. Wiśnieswki, Fano bundles over $\mathbb{P}^{3}$ and $\mathbb{Q}^{3}$, Pacific J. Math., 141 (1990), 197-208, MR 91g:14036, Zbl 0705.14016.

[SW2] _ On Fano manifolds which are $\mathbb{P}^{k}$-bundles over $\mathbb{P}^{2}$, Nagoya Math. J., 120 (1990), 89-101, MR 92e:14038, Zbl 0728.14037. 
[Wi1] J.A. Wiśniewski, Length of extremal rays and generalized adjunction, Math. Z., 200 (1989), 409-427, MR 91e:14032, Zbl 0668.14004.

[Wi2] On Contractions of extremal rays of Fano manifolds, J. Reine Angew. Math., 417 (1991), 141-157, MR 92d:14032, Zbl 0721.14023.

Received May 24, 2001 and revised October 22, 2001.

Dipartimento di Matematica

Università DEgli Studi Di Trento

Via Sommarive 14

I-38050 Povo Trento

ITALY

E-mail address: occhetta@science.unitn.it 